\title{
Structure Tensors for Dispersed Fibers in Soft Materials
}

\author{
Moritz Kalhöfer-Köchling $\odot,{ }^{1,2}$ Eberhard Bodenschatz®, ${ }^{1,2,3,4}$ and Yong Wang ${ }^{1,2,3, *}$ \\ ${ }^{1}$ Max Planck Institute for Dynamics and Self-Organization, 37077 Göttingen, Germany \\ ${ }^{2}$ German Center for Cardiovascular Research, Partner Site Göttingen, 37075 Göttingen, Germany \\ ${ }^{3}$ Institute for Dynamics of Complex Systems, University of Göttingen, 37073 Göttingen, Germany \\ ${ }^{4}$ Laboratory of Atomic and Solid-State Physics and Sibley School of Mechanical and Aerospace Engineering, \\ Cornell University, Ithaca, New York 14853, USA
}

(Received 3 February 2020; revised manuscript received 4 May 2020; accepted 19 May 2020; published 16 June 2020)

Soft tissues, such as skin, myocardium, and chordae tendineae, typically display anisotropic mechanical behavior due to their fibrous nature. In constitutive modeling, fiber families frequently are assumed to be unidirectional. Recent numerical results, however, display the need to incorporate dispersion of fiber orientation. This evidence gets supplemented by new experimental results based on high-resolution second-harmonic imaging microscopy. Generalized structure-tensor (GST) models are frequently utilized to model fiber dispersion, as they are mathematically easy to treat and demand only a little effort to implement. They can be regarded as Taylor-series expansions of the numerically more challenging angular-integration (AI) method, which encompasses a distribution of fiber orientations together with the associated fiber stress. In this work, we show how low-order GST models give rise to numerical instabilities as they show strong sensitivity with regards to the mean fiber orientation. To overcome these instabilities, we propose a different class of GST models, termed squared GST (SGST), which computes faster, is easier to implement, and converges to the AI faster than previous GST models of similar order. The SGST models promise to be adaptable to generalized problems, such as functional decomposition of fiber density as well as coupling between different fiber families. Advanced simulations with the proposed models will shed light on the complex behavior of fiber reinforced soft materials.

DOI: 10.1103/PhysRevApplied.13.064039

\section{INTRODUCTION}

Whether it is neurons, myocytes, or collagen, most soft biological tissues comprise fibers leading to anisotropic mechanical behavior [1,2]. In disease, alteration of mechanical properties of tissues is a valuable biomarker for diagnosis. Since material stiffness is not always accessible directly via state-of-the-art medical measurements, computer simulations are becoming a critical tool in both disease diagnosis and treatment [3-5], necessitating robust numerical and mathematical models to characterize architecture and orientation of fibers.

Evidenced by recent findings [6,7], unidirectional fiber models are insufficient to describe tissue mechanics and thus dispersion of fiber orientation should be included. Concerning constitutive modeling, there are two schools

*yong.wang@ds.mpg.de

Published by the American Physical Society under the terms of the Creative Commons Attribution 4.0 International license. Further distribution of this work must maintain attribution to the author(s) and the published article's title, journal citation, and DOI. Open access publication funded by the Max Planck Society. debating how to treat fiber dispersion mathematically. The first is dating back to Lanir [8], who proposes to attribute an elastic energy to every single fiber, the orientation of which follows a given angular distribution. Then, angular integration (AI) of said energy gives rise to the macroscopic response to external loading. The second school, on the other hand, follows the steps of Gasser et al. [6], who translate the fiber architecture into a generalized structure tensor (GST), which describes collective fiber deformation. Both schools, expectedly, carry their own advantages and disadvantages. The AI furnishes a bottom up, easy to understand energy density while on the other hand, in finite-element simulation, it involves the task of integrating over angle space on each iteration for every single Gauss point, which quickly becomes heavily time consuming. The GST models, in contrast, are easy to implement and fast to simulate since many calculations can be precomputed. In return, exclusion of fibers under compression is difficult [9] but necessary to account for the fact that they usually buckle under compressive loads.

As GST models can be interpreted as low-order Taylorseries expansions of the AI model, the former have been criticized for their lack of accuracy as stated by 
Refs. [10-12]. Conversely, it has been shown by Holzapfel et al. [13] that both models approximate experimental data equally well.

Although both, the GST and the AI, models can be matched to the same experiment, they show large differences in their predictions if small changes in the fiber architecture are introduced. Especially for simple shear the sensitivity to the fiber arrangement is substantial, which is highlighted in Fig. 1. Directional sensitivity, shown in Fig. 1(b), measures how much shear stresses change relatively if the mean fiber orientation is altered. Taking, for example, the $\left(f n_{1}\right)$ shear mode with a shear of $\gamma=0.2$, a dispersionless (HO) and a classical GST model (0GST) deviate in stress by roughly $25 \%$ if the fibers are rotated by only $1^{\circ}$, contrary to the AI model, which varies only by roughly $5 \%$. This behavior becomes even more
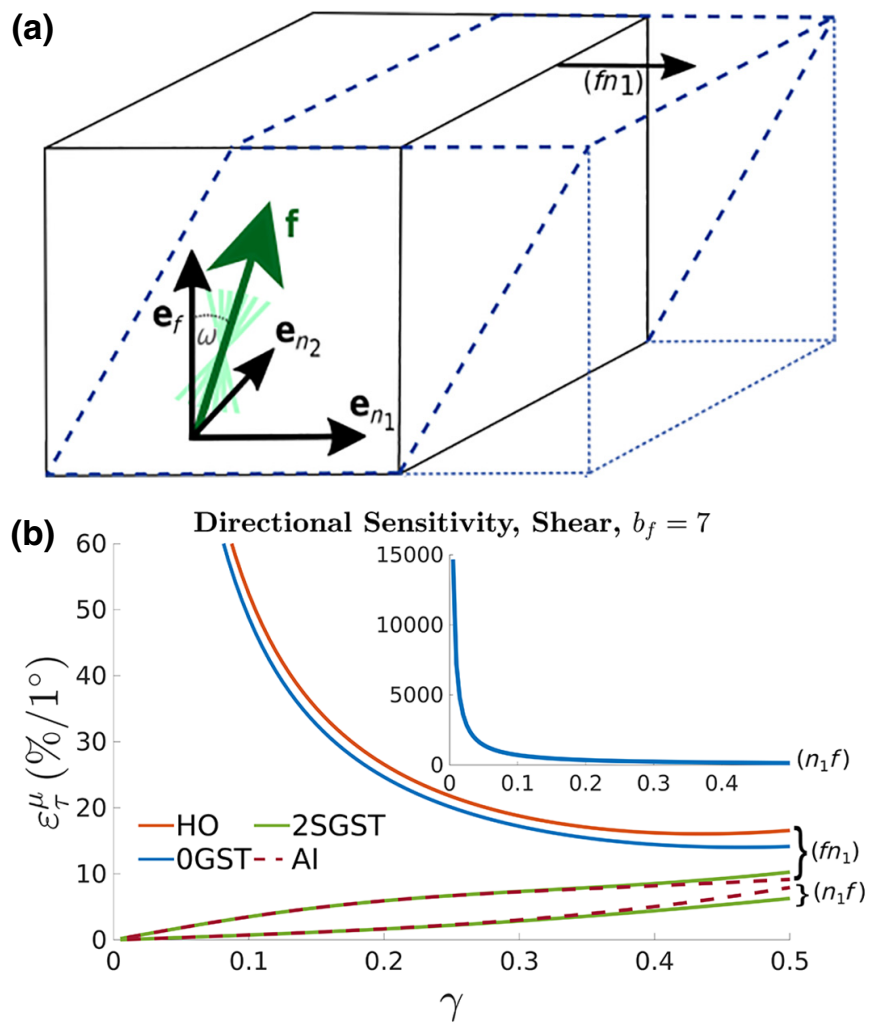

FIG. 1. Directional sensitivity for simple shear. (a) Geometric setup for simple shear with the frame showing original mean fiber orientation ( $\left.\mathbf{e}_{f}\right)$ and two normal directions $\left(\mathbf{e}_{n_{1}}, \mathbf{e}_{n_{2}}\right)$. Unit vector $\mathbf{f}$ portrays the mean fiber direction after rotation $\omega$ in the $\left(f n_{1}\right)$ plane. Solid lines depict the reference configuration versus the deformed one represented by dashed lines. Dotted lines visualize the shear $\gamma$. In (b) the resulting relative change shear stress $\varepsilon_{\tau}^{\mu}$ as defined in Eq. (18) shows that directional sensitivity depends heavily on the degree to which dispersion is embedded in the model ( $\mathrm{HO}>0 \mathrm{GST}>2 \mathrm{GST}>\mathrm{AI}$, cf. Sec. V). All models incorporate the same elastic parameter $b_{f}=7$ and $a_{f}=1$. Shear mode $\left(n_{1} f\right)$ for the 0GST model is shown as an inset while the corresponding value for the HO model diverges. pronounced for smaller shear values or the $\left(n_{1} f\right)$ shear mode.

As we show in this work, directional sensitivity plays a key role in numerical stability even if only small shear deformations are present. While for most ordinary shapes, said sensitivity is negligible, it becomes essential, once realistic conditions with irregular shapes, such as cardiac infarctions or wounds, are considered. To prevent instabilities arising from sensitivities, we develop a class of GST models, termed squared generalized structure-tensor (SGST) models, which closely match the directional sensitivity of the AI model [cf. 2SGST in Fig. 1(b)], while still offering the framework of classical GST models, yielding an easy implementation and rapid numerical calculation. The approach entails the Taylor-series expansion, which shows faster convergence to the AI, and is mathematically easier to implement for higher-order terms, while, at the same time, reducing computational costs compared to conventional GST models of the same order.

The general framework incorporates an exponential fiber strain energy, which has proven successful to describe cardiac [14-16] as well as aortic $[6,17,18]$ tissue in experiment and simulation likewise. The dispersion of fiber angles is governed by the von Mises distribution, which is supported by recent experiments on human myocardium [19] and aortic tissue [20], although our approach can be applied to more general fiber arrangements.

An easy-to-use MATLAB library, comprising the different models up to sixth polynomial order, is provided as Supplemental Material [21].

\section{CONSTITUTIVE MODELING OF DISPEPRSED FIBERS}

Following a commonly used macroscopic framework, we assume that deformation is governed by an affine map $\mathbf{x}=\chi(\mathbf{X})$, which smoothly transforms a material point $\mathbf{X}$ in the stress-free state into the spatial frame $\mathbf{x}$. The deformation gradient, then, is defined as $\mathbf{F}=\partial \mathbf{x} / \partial \mathbf{X}$, with the determinant $J=\operatorname{det}(\mathbf{F})$ measuring local volume changes (incompressibility corresponds to $J \equiv 1$ ). Multiplicative decomposition $\mathbf{F}=\left(J^{1 / 3} \mathbb{I}\right) \overline{\mathbf{F}}$ splits the deformation gradient into a distortional $\overline{\mathbf{F}}$ and a dilational part $J^{1 / 3} \mathbb{I}$. Postulating perfect bonding between ground matrix and fibers, we ignore microscopic nonaffine deformations [22]. The strain of an individual fiber $\mathbf{f}^{\prime}$, thus, follows the simple relation $\epsilon^{2}=\overline{\mathbf{E}}: \mathbf{M}$, with isochoric Green-Lagrange strain $\overline{\mathbf{E}}$ and structure tensor $\mathbf{M}$ defined as

$$
\overline{\mathbf{E}}=\frac{1}{2}\left(\overline{\mathbf{F}}^{\mathrm{T}} \overline{\mathbf{F}}-\mathbb{I}\right), \quad \mathbf{M}=\mathbf{f}^{\prime} \otimes \mathbf{f}^{\prime},
$$

where $\mathbb{I}$ is the identity tensor. The AI for an arbitrary function $g$ is defined as the average $\langle\cdot\rangle$ over the solid 
angle [8]

$$
\langle g\rangle=\frac{N_{\text {fiber }}}{4 \pi} \int_{0}^{\pi} \int_{0}^{2 \pi} \rho\left[\mathbf{f}^{\prime}(\Theta, \phi)\right] g(\Theta, \phi) \sin \Theta d \phi d \Theta,
$$

where the fiber density per volume $N_{\text {fiber }}=1$ w.l.o.g. and $\rho\left[\mathbf{f}^{\prime}(\Theta, \phi)\right]$ is the fiber density per solid angle $d \Omega=$ $\sin \theta d \phi d \theta$. Assuming an energy density $\psi_{f}(\epsilon)$ of an individual fiber, the AI yields the total fiber energy density

$$
\Psi_{f}=\left\langle\psi_{f}(\epsilon)\right\rangle
$$

As it has been used successfully to describe, for example, mammalian myocardium (human [19] and dog [23]) as well as arterial walls [6], we focus our attention on the von Mises distribution

$$
\rho(\Theta)=4 \sqrt{\frac{b_{\rho}}{2 \pi}} \frac{\exp \left(b_{\rho}[\cos (2 \Theta)+1]\right)}{\operatorname{erfi}\left(\sqrt{2 b_{\rho}}\right)},
$$

which is controlled through dispersion coefficient $b_{\rho}$. It is centered about $\Theta=0$ representing the mean fiber orientation $\mathbf{e}_{f}$ and shows rotational symmetry. The latter attribute is chosen for convenience, although the following analysis can be applied to more general, asymmetric distributions. For biological fibers, exponential stiffening is most commonly observed and can be expressed using [17]

$$
\psi_{f}(\epsilon)=\frac{a_{f}}{2 b_{f}}\left(e^{4 b_{f} \epsilon^{4}}-1\right)
$$

where $a_{f}$ and $b_{f}$ comprise linear and nonlinear elastic material properties. Using finite-element methods (FEM) to simulate such material requires repetitive numerical calculation of Eq. (2) on each time step for every single Gauss point, thus making it heavily time consuming. Contrary to the exponential energy function shown in Eq. (5), trigonometric functions can easily be integrated, inspiring the use of GST. Classically, the GST $\mathbf{H}$ is defined as [6]

$$
\mathbf{H}=\langle\mathbf{M}\rangle=\kappa \mathbb{I}+(1-3 \kappa) \mathbf{M}(\Theta=0, \phi=0),
$$

together with structure parameter $\kappa$

$$
\kappa=\frac{1}{2}\left\langle\sin ^{2}(\theta)\right\rangle,
$$

which, in three dimensions, is confined to the interval $[0,1 / 3]$, where 0 reflects unidirectional behavior while $1 / 3$ embodies complete isotropy. Following an approach similar to Ref. [11], $\mathbf{H}$ can be used as an anchor point for (a)

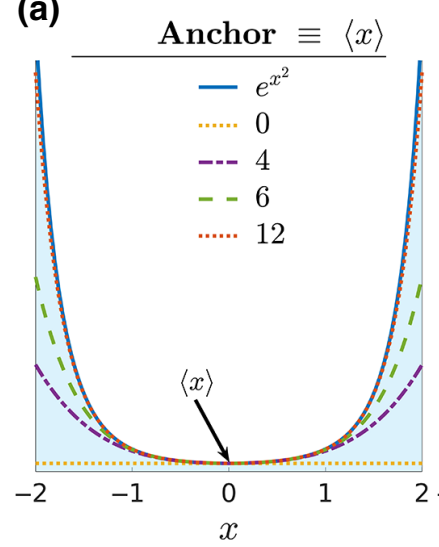

(b)

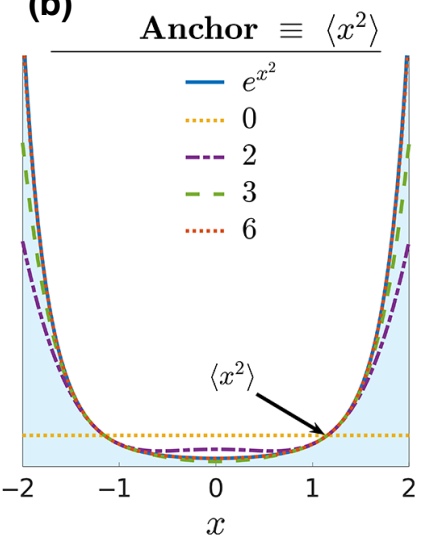

FIG. 2. Taylor series of $f(x)=e^{x^{2}}$ performed for two different anchor points. The numerals in the respective legends signify the order to which the Taylor series is performed. If this number, in the case of (b), is multiplied by 2 the product matches the polynomial order in $x$ of (a). The area shaded in light blue signifies the integral to be approximated.

a Taylor-series expansion of Eq. (2). Using multi-index notation, the $n$ th-order approximation is defined as

$$
\Psi_{f}^{n \mathrm{GST}}=\sum_{|\alpha| \leq n} \frac{D^{\alpha} \psi_{f}\left(\left\langle\epsilon^{2}\right\rangle\right)}{\alpha !}\left\langle(\mathbf{M}-\mathbf{H})^{\alpha}\right\rangle
$$

The zeroth-order term, consequently, reads

$$
\Psi_{f}^{0 \mathrm{GST}}=\frac{a_{f}}{2 b_{f}} e^{4 b_{f}(\mathbf{E}: \mathbf{H})^{2}}=\psi_{f}\left(\left\langle\epsilon^{2}\right\rangle^{2}\right)
$$

Using Jensen's inequality, however, we find that

$$
\left\langle\psi_{f}\left(\epsilon^{4}\right)\right\rangle \geq \psi_{f}\left(\left\langle\epsilon^{4}\right\rangle\right) \geq \psi_{f}\left(\left\langle\epsilon^{2}\right\rangle^{2}\right)
$$

which sparks the idea that $\left\langle\epsilon^{4}\right\rangle$ promises to be better suited as an anchor point for the Taylor expansion, as we later confirm in Sec. III. The effects of this paradigm shift are showcased in Fig. 2, where the averages of two different Taylor series of the function $f$

$$
\langle f(x)\rangle=\left\langle e^{x^{2}}\right\rangle=\frac{\sqrt{\pi}}{4} \operatorname{erfi}(2)
$$

are compared. For demonstration purposes, here, we focus on the simple case of a one-dimensional function and the average $\langle\cdot\rangle$ performed with respect to a homogeneous probability density on the interval $[-2,2]$. It is evident that anchor point $\left\langle x^{2}\right\rangle$ shows faster convergence, and closer proximity to Eq. (11), which is highlighted for small orders in $x$ in Table I. 
TABLE I. Taylor series of Eq. (11) for two different anchor points $\left(\langle x\rangle\right.$ and $\left.\left\langle x^{2}\right\rangle\right)$ and different polynomial orders $n_{x}$.

\begin{tabular}{lcccccccc}
\hline \hline$n_{x}$ & 0 & 2 & 4 & 6 & 8 & 10 & 12 & $\infty$ \\
\hline$\langle x\rangle$ & 1 & $2 . \overline{3}$ & $3.9 \overline{3}$ & 5.46 & 6.64 & 7.42 & 7.86 & 8.23 \\
$\left\langle x^{2}\right\rangle$ & 3.79 & 3.79 & 6.491 & 7.18 & 7.86 & 8.08 & 8.18 & \\
\hline \hline
\end{tabular}

We, therefore, define the squared generalized structure tensor

$$
\mathbf{H}_{\mathbf{2}}=\langle\mathbf{M} \otimes \mathbf{M}\rangle
$$

which yields a corresponding class of fiber-dispersion models

$$
\Psi_{f}^{n \mathrm{SGST}}=\sum_{|\alpha| \leq n} \frac{D^{\alpha} \psi_{f}\left(\left\langle\epsilon^{4}\right\rangle\right)}{\alpha !}\left\langle\left(\mathbf{M} \otimes \mathbf{M}-\mathbf{H}_{\mathbf{2}}\right)^{\alpha}\right\rangle,
$$

which computes faster, approximates the AI model more closely, and is mathematically easier to handle than GST models of identical order in $\epsilon^{2}$. All structure tensors needed for a Taylor expansion up to second order can be found in the Appendix together with Eq. (6).

\section{PROXIMITY ANALYSIS}

In the following, we quantify the relation between AI, GST, and SGST models analytically through variations in stresses. Therefore, we adopt fiber stress $\overline{\boldsymbol{\sigma}}^{\mu}$ for an incompressible material [16]

$$
\overline{\boldsymbol{\sigma}}^{\mu}=\mathbf{F} \frac{\partial \Psi_{f}^{\mu}}{\partial \mathbf{E}} \mathbf{F}^{T}-p(J) \mathbb{I}
$$

where $\mu \in\{2 \mathrm{GST}, 4 \mathrm{GST}, 0 \mathrm{SGST}, 2 \mathrm{SGST}\}$ specifies the model of interest, and $p(J)$ is the Lagrangian multiplyer function conserving incompressibility. We confine our analysis to the loading protocol $\mathbb{T}$ comprising biaxial stretch and simple shear. As depicted in Fig. 1, model predictions heavily rely on fiber architecture, wherefore $\mathbb{T}$ includes fibers rotated in $\left(f n_{1}\right)$ plane by angle $\omega$. Together with the different biaxial stretch ratios $\beta$ we consider the following loading protocols:

$$
\begin{gathered}
\mathbb{T}_{\text {shear }}=\left[\left(f n_{1}, \omega\right),\left(n_{1} f, \omega\right),\left(n_{1} n_{2}, \omega\right) \mid \omega \in\left(0^{\circ}, 45^{\circ}\right)\right], \\
\mathbb{T}_{\text {biax }}=\left\{(f f, \omega, \beta),\left(n_{1} n_{1}, \omega, \beta\right) \mid \omega \in\left(0^{\circ}, 45^{\circ}\right)\right. \\
\wedge \beta \in\left[\left(1: \frac{1}{2}\right),\left(1: \frac{3}{4}\right),(1: 1),\right. \\
\left.\left.\left(1: \frac{4}{3}\right),(1: 2)\right]\right\} .
\end{gathered}
$$

The choice for the exponent $b_{f}$, which defines the nonlinear elastic fiber response, is loosely based on a parameter estimation attributed to the AI for experimental data for human hearts acquired by Sommer et al. [15,19].

As a metric of accuracy for a single realization of fiber distribution belonging to dispersion coefficient $\kappa \sim b_{\rho}$ [see Eq. (7)], we define the local relative error (LRE)

$$
\Delta_{\kappa}^{\mathrm{LRE}, \mu}(\mathbb{T}, \gamma)=\sqrt{\sum_{t \in \mathbb{T}}\left(1-\frac{\sigma_{t}^{\mu}(\gamma)}{\sigma_{t}^{\mathrm{AI}}(\gamma)}\right)^{2} /\|\mathbb{T}\|,}
$$

where $\gamma$ describes the current shear or stretch depending on the loading protocol, and the normalization coefficient $\|\mid \mathbb{T}\|$ denotes the cardinality of the set of experiments. Models corresponding to first-order terms are not listed since they are equivalent to the zeroth-order terms. Building on the local error estimate, we also define an accumulated or averaged error. It serves as an estimate for the accountability of a given model for a specific choice of $\kappa$. As such, we define the averaged relative error (ARE)

$$
\Delta^{\mathrm{ARE}, \mu}(\mathbb{T}, \kappa)=\sqrt{\int_{\gamma_{\min }}^{\gamma_{\max }} \frac{\Delta_{\kappa}^{\mathrm{LRE}, \mu}(\mathbb{T}, \gamma)^{2}}{\gamma_{\max }-\gamma_{\min }} d \gamma,}
$$

with $\left(\gamma_{\min }, \gamma_{\max }\right)=(0,0.5)$ for simple shear and $\left(\gamma_{\min }, \gamma_{\max }\right)$ $=(1,1.1)$ for biaxial stretch.

The resultant proximity estimates are displayed in Fig. 3. Therein, plots (a) and (d) display how accurately the different models match the AI for a dispersion coefficient $\kappa=0.06$, which is taken from Ref. [19]. The corresponding LRE plotted in the second column for the same choice of parameters as in the first column, are consulted for better comparability. The double logarithmic plot of the LRE shows nicely how different orders of approximation scale linearly with the deformation coefficient $\gamma$. It becomes apparent that the accuracy of the 2GST is related to 0SGST while 4GST is related to 2SGST in their approximation behavior. In the case of $\kappa=0.06$ we calculate the AI model with numerical schemes offered by Mathematica, where we chose an accuracy of 16 digits. As this numerical accuracy is exhausted, further numerical errors impact the LRE, which explains the little kinks in Fig. 3(b), which emerge as $\varepsilon_{\tau}^{2 \mathrm{SGST} / 4 \mathrm{GST}} \rightarrow 10^{-7}$.

It has to be remarked, that for biaxial tests, the 4GST model shows much larger relative errors than the 2SGST. Eventually, these errors even exceed those of the 0SGST and 2GST models as $\gamma$ increases, which is a natural result of the different scaling. As large stretch values dominate the ARE, we can observe corresponding peaks at approximately $\kappa=0.1$ in Figs. 3(c) and 3(f), heavily suggesting not to use the 4GST model for the chosen range of deformation $\gamma$. Conversely, we find that in all categories, the 2SGST model shows lowest errors within the 


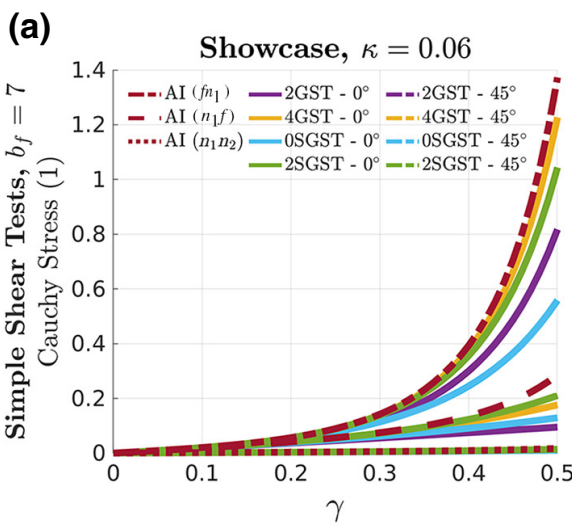

(d)

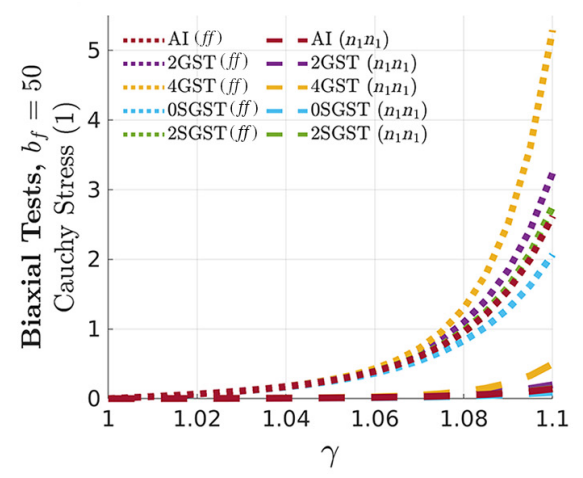

(b)

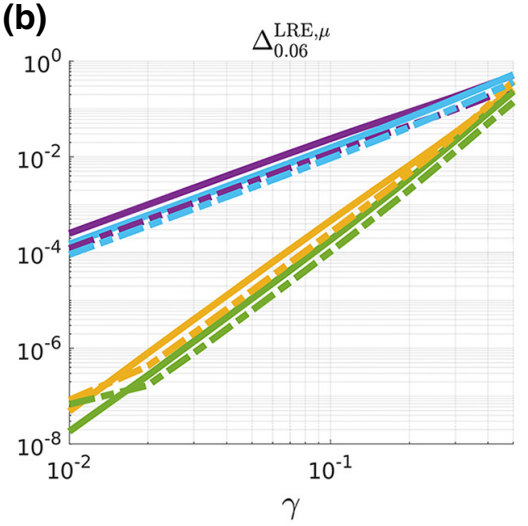

(e)

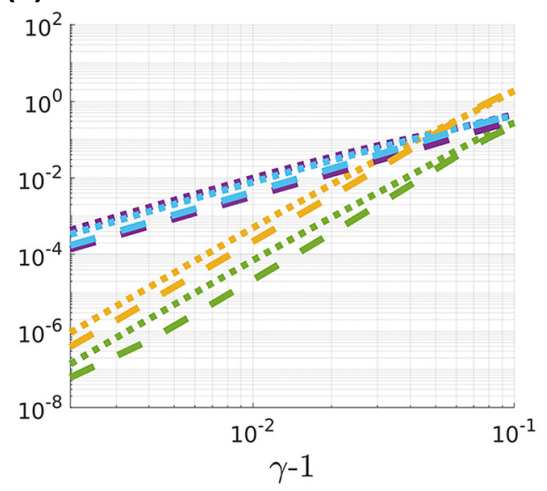

(c)

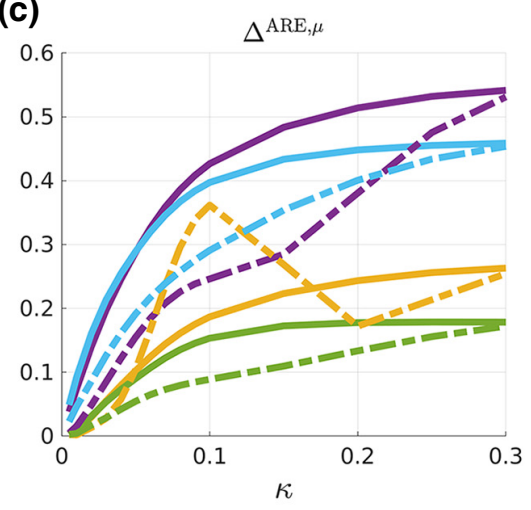

(f)

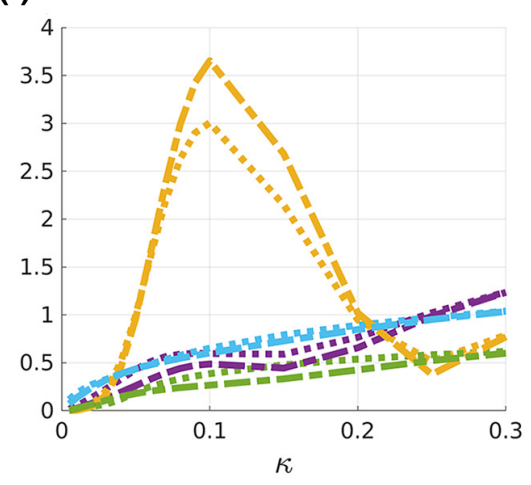

FIG. 3. (a)-(c) Proximity analysis for the two different model classes $n$ GST [Eq. (8)] and $n$ SGST [Eq. (13)] to the AI [Eq. (2)] in the case of simple shear with $a_{f}=1$ and $b_{f}=7$. They share a common legend from (a). (d)-(f) The same analysis for biaxial stretch, where $b_{f}=50$. They share a common legend from (d). (a),(d) Representative Cauchy stress for simple shear and equibiaxial stretch, respectively, with $\kappa=0.06$, which can be interpreted as $20 \%$ isotropy. (b),(e) Local relative errors $\Delta_{\kappa}^{\mathrm{LRE}, \mu}$ [Eq. (16)] with all parameters chosen as in (a),(d). The logarithmic scale reveals at which power in shear or strain the AI is approximated. (c),(f) are concerned with the accumulated relative error $\Delta^{\mathrm{ARE}, \mu}$ [Eq. (17)] for different degrees of dispersion, ranging from unidirectional $(\kappa=0)$ to complete isotropic $(\kappa=1 / 3)$ behavior. Since the $\left(n_{1} n_{2}\right)$ mode for shear and the $\left(n_{1} n_{1}\right)$ mode for biaxial stretch yield relatively small stresses, the different models overlap in plots (a),(c).

boundaries considered, making it a promising candidate for simulation.

With a fixed dispersion coefficient $\kappa$ we can analyze how the ARE changes as the polynomial order $n$ gets further increased. Figure 4 confirms the counterintuitive increase of the $\Delta^{\mathrm{ARE}, n \mathrm{GST}}$ with $\kappa=0.06$ as discussed above. For biaxial tests and simple shear tests incorporating fibers rotated by $45^{\circ}$ in the $\left(f n_{1}\right)$ plane this tendency even proceeds beyond $n=10$, thus discouraging the use of $n \mathrm{GST}$ models as an approximation to the AI. For the corresponding $n / 2$ SGST models depicted, on the other hand, the ARE shows a desired decreasing tendency. Of course this picture is by no means complete and different loading protocols can lead to strikingly divergent error estimates, both for the GST and SGST model classes. Hence, a careful consideration of the occurring loading protocols together with the desired accuracy should shed light on the viability of a given approximation scheme before simulation.

\section{NUMERICAL EFFICIENCY}

Quantifying numerical efficency is important to evaluate advantages and disadvantages of an approximation scheme. Nonetheless, finding an appropriate metric for the numerical efficiency of the AI model is difficult as it depends not only on the quadrature rule, and precision requirements used, but it is also heavily influenced by the deformation gradient $\mathbf{F}$ as well as the dispersion coefficient $b_{\rho}$. For best comparability with the results presented in Secs. III and V, we constrain our analysis to simple shear and equibiaxial tests, examining the same range of shear and extension values $\gamma$ as there.

In order to keep the efficiency estimation independent of any additional algorithms used in a FEM solver, we restrain it to algebraic computation times of the individual AI, SGST, and GST expressions. The resulting computation times can be found in Fig. 5. All calculations are performed with Mathematica on a Windows 10 


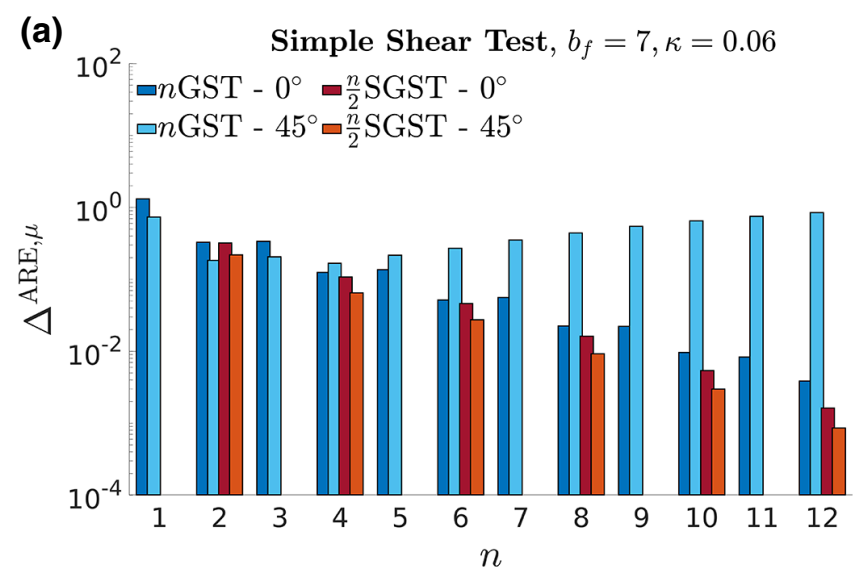

(b)

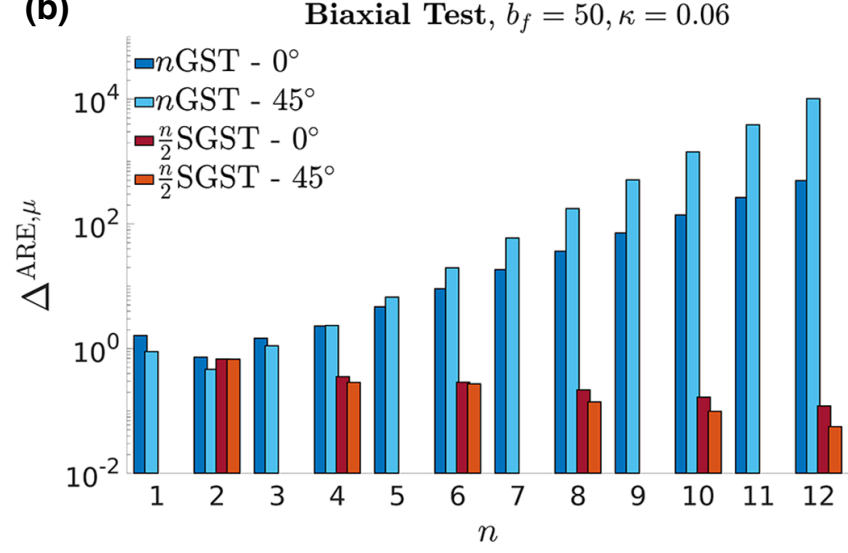

FIG. 4. Averaged relative error as defined in Eq. (17) for different polynomial orders $n$ similar as in Figs. 3(c) and 3(e) with constant dispersion coefficient $\kappa$. Clearly, an increase of $n$ does not imply smaller errors for the GST model, contrasting the continuous decline for the SGST model.

computer with an Intel $^{\circledR}$ Core $^{\mathrm{TM}}$ i5-7400 CPU and 8GB of memory. For the AI model we utilize an inbuilt global adaptive quadrature rule employing a working precision of eight decimals.

As shown in Fig. 5 the computation time increases exponentially with the order to which the Taylor series is performed. For identical values of $n \in\{2,4, \ldots, 12\}$, the $n$ GST model takes about $25-180 \%$ more time compared with the $n / 2$ SGST model. This result gets supplemented by calculations performed with our MATLAB library [21], where 4GST takes roughly $25 \%$ more computing time compared with its counterpart 2SGST. Such a trend can be attributed to the overall larger algebraic complexity of $n \mathrm{GST}$ polynomials. Beyond an order of $n=10$ the prevalence of the $n / 2 \mathrm{SGST}$ model class over the AI becomes questionable, as shown in Fig. 5.

\section{DIRECTIONAL SENSITIVITY}

When measuring the mean fiber orientation of a given specimen experimentally, such a task can be very difficult,

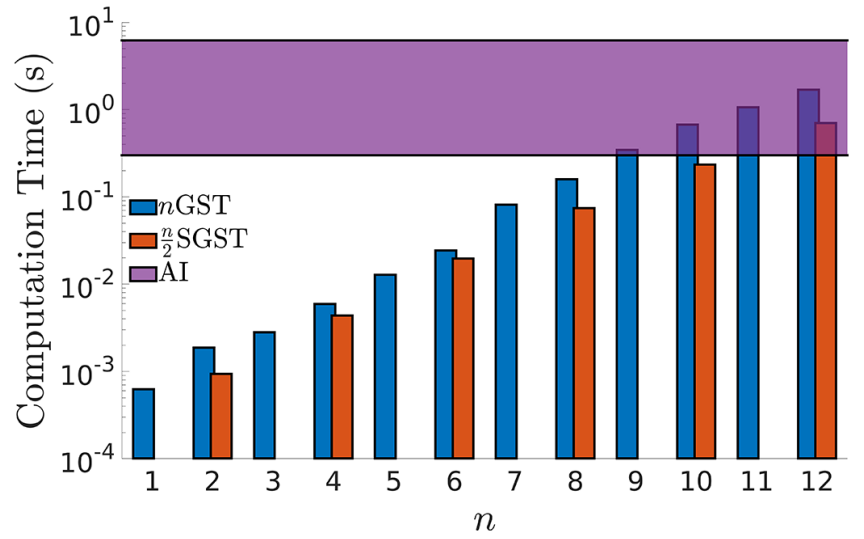

FIG. 5. Algebraic computation times for individual realizations of the GST and SGST model classes, grouped with regards to similar error scaling as presented in Figs. 3(b) and 3(e). The purple region represents the domain of observed computation times for the AI with a precision of eight digits.

not only because fibers in reality can show strong dispersion, but also because for most tissues this mean fiber orientation is not constant on scales, which stress measurements are performed on. If the model used shows strong dependence on the actual fiber orientation, systematic measurement errors are amplified. As a result, when choosing a model to match experimental results, not only good statistical agreement, but also small systematic errors should be accounted for.

In the case of simple shear, fibers initially are aligned with the cube's perimeters as graphed in Fig. 1(a). As before, let $\omega$ denote the angle between the assumed and the true mean fiber orientation in the $\left(f n_{1}\right)$ plane, then, the relative change in predicted stress for a given model $\mu$ is computed via

$$
\varepsilon_{\tau}^{\mu}=\frac{1}{\bar{\sigma}_{\tau}^{\mu}} \frac{\partial \bar{\sigma}_{\tau}^{\mu}}{\partial \omega},
$$

where $\tau \in \mathbb{T}$. When there is no dispersion $(\mu=\mathrm{HO}$, as proposed by Holzapfel and Ogden [16]), i.e., when $\kappa=0$, Eq. (18) can be calculated analytically

$$
\varepsilon_{\tau}^{\mathrm{HO}}(\gamma)= \begin{cases}\frac{3+4 b_{f} \gamma^{4}}{\gamma} & \tau=\left(f n_{1}\right) \\ \infty & \tau \in\left\{\left(n_{1} f\right),\left(n_{1} n_{2}\right)\right\} \\ 0 & \text { else }\end{cases}
$$

in the case of simple shear. Figure 1(b) shows how directional sensitivity $\varepsilon_{\tau}^{\mathrm{HO}}$ diverges for several different loads, which can lead to undetermined fiber orientations in simulation.

Clearly, dispersion decreases this sensitivity, since such an arrangement supports loads of different directions. Consequently, studies investigating the dependency of local 
stresses on the fiber architecture, like Refs. [24] and [25] for the left ventricle, might rather express dependence on the actual mathematical model used. However, this dependence not only affects error estimates for material properties but also highly influences numerical stability as pointed out by Duong [26].

Numerical stability depends on a multitude of factors, starting off from the physics through the actual solver scheme to the the mesh quality. Controlling all these influences, and factoring out under which conditions instabilities are a major concern, is difficult. Instead of controlling the quality of a simulation through mesh size and error tolerance we focus on the influence of the order to which dispersion is captured in a given model. Thus, we focus on a scenario that reveals checkerboard instabilities for a dispersion-free model. Although the scenario, demonstrated below, is rather specific, its key features are applicable to a wide range of problems.

To compare the stability of different models, we match them to the same loading protocol. Next, we identify another loading condition, which displays directional sensitivity. Finally, we construct a simulation that is dominantly governed by the former protocol, yet is superposed with the latter. As shown in Fig. 1(b), both the HO and 0GST model, show strong directional sensitivity with respect to shear load. Thus, we match biaxial load and simulate a monoaxial setup, as depicted in Fig. 6, which exerts increased shear due to its different constituent materials A and B. Material B is governed by an isotropic Neo-Hookean strain energy density

$$
\Psi_{\text {isovol }}^{B}=C_{\mathrm{Hook}} \overline{\mathbf{E}}: \mathbb{I},
$$

with material coefficient $C_{\text {Hook }}$ together with the shape of B chosen such that, during tensile loading, torque is exerted. Material A on the other hand is a ground matrix-fiber composite, following the constitutive law

$$
\Psi_{\text {isovol }}^{A}=\frac{a}{2 b} e^{4 b \overline{\mathbf{E}}: \mathbb{I}}+\Psi_{f}^{\mu}(\overline{\mathbf{E}})
$$

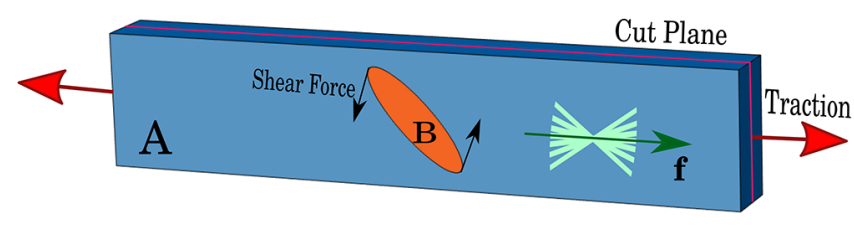

FIG. 6. A beam, composed of two different materials A and $\mathrm{B}$, is subject to monoaxial stretching. Material A consists of fibers with mean direction pointing along $\mathbf{f}$, supported by a soft isotropic ground matrix. Material B on the other hand is purely isotropic and chosen to be stiff enough to give rise to strong shear forces (cf. Table II). Lines in magenta show the cut plane used for visualization. with the different models $\mu \in\{$ HO,0GST,2SGST $\}$. In simulation, it is easiest to comply closely with incompressibility by employing a volumetric strain energy $\Psi_{\mathrm{vol}}(J)$ to penalize volume changes. Here we chose [27]

$$
\Psi_{\mathrm{vol}}=\Gamma\left[\left(J^{2}-1\right) / 2-\log J\right],
$$

with bulk modulus $\Gamma=100 \mathrm{MPa}$. The total energy density

$$
\Psi_{\mathrm{tot}}^{A / B}=\Psi_{\mathrm{vol}}(J)+\Psi_{\text {isovol }}^{A / B}(\overline{\mathbf{E}}),
$$

then, leads to Cauchy stress

$$
\boldsymbol{\sigma}^{\mu}=\mathbf{F} \frac{\partial \Psi_{\text {tot }}^{A / B}}{\partial \mathbf{E}} \mathbf{F}^{T}
$$

and von Mises stress

$$
\left(\sigma_{\text {Mises }}^{\mu}\right)^{2}=\frac{3}{2}\left\{\operatorname{Tr}\left[\left(\sigma^{\mu}\right)^{2}\right]-\operatorname{Tr}^{2}\left(\boldsymbol{\sigma}^{\mu}\right)\right\} .
$$

Parameters for the different isovolumetric energies of material A are listed in Table II and are used together with $C_{\text {Hook }}=40 \mathrm{MPa}$. These parameters are obtained from minimizing the Chi square

$$
\chi_{\mu}^{2}=\sum_{\tau \in \mathbb{T}_{\text {equi }}} \sum_{i=0}^{N}\left[\sigma_{\tau}^{\mu}(1+i \Delta \gamma)-\sigma_{\tau}^{2 \mathrm{SGST}}(1+i \Delta \gamma)\right]^{2}
$$

for the two equibiaxial stresses $\mathbb{T}_{\text {equi }}=\left\{\left(f f, 0^{\circ}, 1\right)\right.$, $\left.\left(n_{1} n_{1}, 0^{\circ}, 1\right)\right\}$ with corresponding discretized stretches $\Delta \gamma=0.22 / N$ and $N=22$. The relative difference

$$
\delta^{\mu}(\gamma)=\left|\frac{\sigma_{\tau}^{\mu}-\sigma_{\tau}^{2 \mathrm{SGST}}}{\sigma_{\tau}^{2 \mathrm{SGST}}}\right|
$$

for the resulting set of parameters is portrayed in Fig. 7(a). If, in contrast, this change of variables is not performed, and, instead, the same set of parameters is used for all three models, directional sensitivity becomes pronounced even more strongly. However, even if equibiaxial loading is matched to high degree, directional sensitivity analysis shows that sensitivity is lowest for the 2SGST. From

TABLE II. Monoaxial stretch parameters, corresponding to Eq. (21) after models are matched for equibiaxial stretch as shown in Fig. 7(a).

\begin{tabular}{lccccc}
\hline \hline Model & $a(\mathrm{kPa})$ & $b$ & $a_{f}(\mathrm{kPa})$ & $b_{f}$ & $b_{\rho}$ \\
\hline 2SGST & 1.5 & 1 & 7 & 40 & 4.5 \\
HO & 0.0363 & 26.9 & 4.65 & 38.1 & 0 \\
0GST & 0.0183 & 24.6 & 8.82 & 48.43 & 4.5 \\
\hline \hline
\end{tabular}



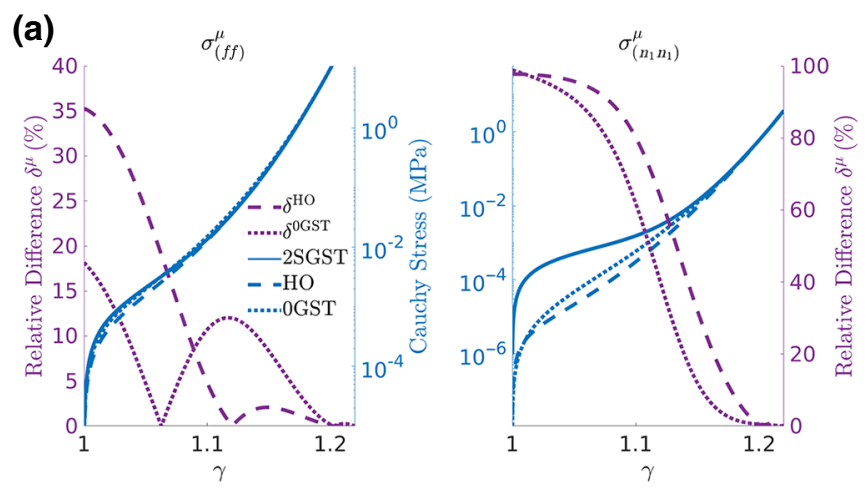

(b)

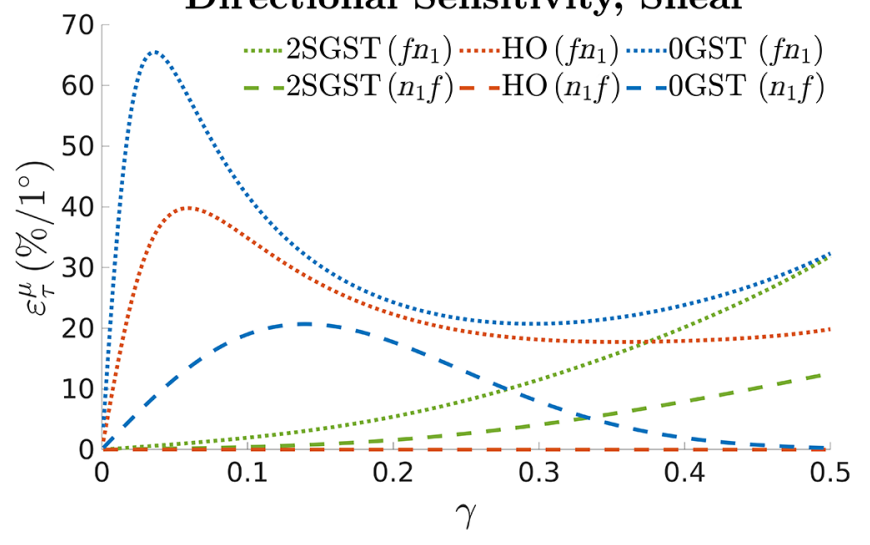

FIG. 7. (a) shows how well the HO and 0GST models match the 2SGST model for equibiaxial stretch after fitting. Corresponding parameters can be found in Table II. True stresses are plotted in blue, whereas relative differences are depicted in purple. (b) Directional sensitivity for the same parameters as in (a) in the case of simple shear.

Fig. 7(b) it can be deduced that, for example, at $\gamma=0.1$ directional sensitivities $\varepsilon_{\left(f n_{1}\right)}^{\mu}$ of the HO and 0GST models are roughly ten times larger than that of the 2SGST model. As shear increases, the relation changes qualitatively and $\varepsilon_{\left(f n_{1}\right)}^{2 \mathrm{SGST}}>\varepsilon_{\left(f n_{1}\right)}^{\mathrm{HO}}$, which can be attributed to the strong nonlinearity in isotropic energy $(b=26.9)$. Also the bivariate nature of the 0GST is revealed, approaching $\varepsilon_{f n_{1}}^{2 \text { SGST }}$ on the one hand, and $\varepsilon_{n_{1} f}^{\mathrm{HO}}$ on the other hand as $\gamma$ increases.

Surely this sensitivity alone is not capable of predicting stable or unstable behavior, far and foremost because actual simple shear is not present in this simulation. Yet, it nicely motivates the appearance of jigsaw patterns, which can be observed in the von Mises stress defined in Eq. (25) and pictured in Fig. 8. It can be observed that the strength of these patterns decreases from HO, over 0GST to 2SGST. Moreover, the algorithm did not converge for the HO model beyond a stretch of $\gamma-1=19 \%$. Also the 0GST model ceased to converge beyond $15 \%$ if the ramping is not reduced from $3 \%$ to $1 \%$ per step. At the same time, the 2SGST showed no irregular behavior for stretches up

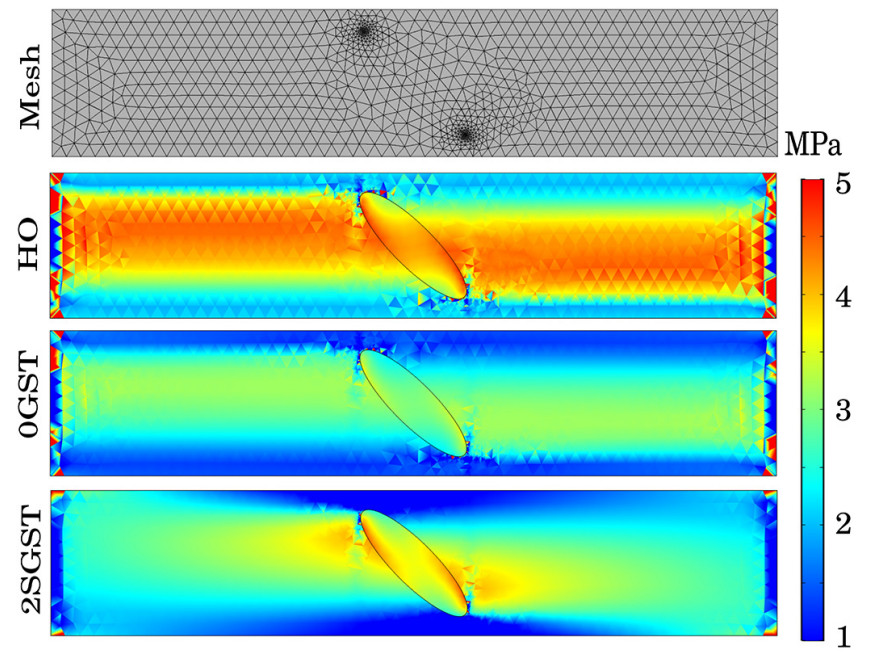

FIG. 8. von Mises stress as defined in Eq. (25) reveals directional instability in monoaxial stretch simulation for the $\mathrm{HO}$, 0GST, and 2SGST models in the cut plane mentioned in Fig. 6. Contrary to the 2SGST model, HO shows a clear checkerboard pattern, which is a namesake for the instability that arises from directional sensitivity. The 0GST model shows intermediate behavior as expected. The result is shown in the reference frame for $18 \%$ stretch. The numerical scheme no longer converges for stretches above $19 \%$ in the case of HO. In the top subfigure the three-dimensional (3D) mesh projected onto the cut plane is plotted. It should be noted that the checkerboard patterns at the left and right boundaries are due to the numeric scheme. In the case of internal angles below $90^{\circ}$ such singularities are a common feature in FEM simulations.

to $30 \%$. At stretches this large, incompressibility no longer holds with the chosen bulk modulus and the parameters are not fine tuned to match equibiaxial stretch beyond $\gamma-1=22 \%$, prohibiting further comparison between the models.

All simulations are performed using COMSOL Multiphysics version 5.4 with FEM solver PARDISO, assuring a relative error tolerance of $10^{-3}$. Variables to be solved for are fully coupled with an automatic nonlinear Newton method. The mesh contains approximately 20000 prisms, which, together with cubic serendipidity interpolation functions, amount to a total of approximately 450000 degrees of freedom. Tensile load is initially ramped by $3 \%$ per step, which later gets supplemented by a $1 \%$ increase per step as instabilities arise.

\section{DISCUSSION AND CONCLUSIONS}

Considering Jensen's inequality sparked the idea to use a squared, instead of linear, GST as an anchor point of the Taylor series of the AI. Convergence analysis, then, shows reduced errors for the SGST models compared to their 
GST counterpart of same polynomial order, when considering simple shear and biaxial stretch. As fiber architecture has a strong influence on material mechanics, said analysis contains, both fibers aligned with principle directions of the deformation, as well as fibers rotated by $45^{\circ}$ in the respective plane.

Evidently, depending on the extent of deformation, the 2SGST model makes for a reliable substitute of the AI in simulations. Although, in principle, Taylor expansions can be taken to an arbitrary order, since we are dealing with multivariate expressions, they quickly increase in computational costs as demonstrated in Sec. IV. This effect gets weakened by the $n$ SGST models, because the amount of summands does not grow as fast as for the $n \mathrm{GST}$ models. Nontheless, if $n$ gets increased, a complementary increase in accuracy is quickly weighed out by computation time. As a result, if better accuracy is needed, the 2SGST, instead, might be used in conjunction with additive decomposition of the fiber density $\rho$ as detailed in Refs. [7] and [28]. Consequently, also nonaffine deformations, crimping and buckling [29,30] or reorientation [31] of fibers, can be included, which is by default not possible for an approach using Taylor series only.

Sensitivity analysis, on the other hand, reveals how dispersion models deviate from unidirectional ones. Although Holzapfel et al. [13] showed that the 0GST and AI can be matched equally well to equibiaxial and uniaxial experiments on aortic tissue, it becomes evident that these models no longer show the same behavior once fibers are rotated. Further, said sensitivity can lead to checkerboard instabilities during simulation if multiple loading conditions are superposed. A good recipe for instabilities involves strong directional sensitivity, which might be due to a weak ground matrix, plus material inhomogeneities breaking the symmetry of the loading protocol. These conditions can be found, for example, in cardiac tissue involving irregular, infarcted regions, which get even reinforced by strong stresses during systole. Also materials displaying pronounced gradients in their fiber architecture, such as the ventricular heart wall or arteries, might be vulnerable to directional sensitivity.

The advise to use dispersion in order to overcome instabilities is to be taken with a pinch of salt, though, as numerical stability, in principle, should not decide over what kind of physics to implement. Consequently, instead, experiments have to be consulted to rule out the actual directional sensitivity of a given material. This factors in, especially, when quantitative, patient-specific studies are conducted. Further, we want to highlight that simulations are only a viable tool in predicting material behavior, for as long as the loading protocols encountered in computations are validated in experiments. Admittedly, when choosing our model parameters, we violate this key prerequisite, as we consider only biaxial stretch as a reference for the HO, 0GST, and 2SGST model, although in simulation of monoaxial stretch, a convoluted superposition of shear and stretch can be observed. Consequently, different numerical stability and altered stress fields are observed, indicating that a larger set of reference data is needed to properly inform simulation. It has been noted in Ref. [32] that the amount of independent tests, needed to fully describe a material is at least equal to the amount of invariants associated with the material. Since different orders $n$ are associated with qualitatively different $n$ SGST models, we want to remark that even more independent experiments are needed to make an accurate choice of $n$. If the required amount of independent experiments is not met, we advice to use the model with lowest sensitivity with respect to possible sources of errors, like for example the mean fiber orientation.

We want to emphasize, that, although a specific fiber density, namely the symmetric von Mises distribution, is employed in this work, the principle idea behind SGST models can be extended to other, e.g., nonsymmetric, distributions analogously [20,29,33]. Also cross terms between different fiber families as detailed by Melnik et al. [34] show the potential to benefit from an SGST approach.

Characterizing the architecture and orientation of fibers is crucial to predict the mechanical response of soft materials. As the SGST models can be used for fast and robust simulations, they offer likewise robust and noninvasive numerical tool for clinical treatment. One of their applications is to guide patient care in cardiac ischemia. As demonstrated by Fomovsky et al. [35,36], fiber architecture is essential when designing therapeutic implants. Our further work focuses on utilization of the SGST models for in silico experiments to optimize engineered tissue scaffolds [37,38] and hence instruct personalized solutions for recovering pump function of the patient's heart. Another potential application for SGST models is the estimation of peak stresses, which goes hand in hand with the directional sensitivity of the model of choice. Consequently, this choice must be made with care, for example, in the analysis of rupture in aortic aneurysm [39], or atherosclerotic plaque [40]. Likewise, the load-bearing capacity of tendons and ligaments can be studied and optimized [30,41] using the proposed models.

\section{ACKNOWLEDGMENTS}

The authors thank Alain Pumir, Wolfram-Hubertus Zimmermann and Gerhard Holzapfel for their advice and fruitful discussions. This work is supported by the Max Planck Society and the German Center for Cardiovascular Research.

\section{APPENDIX: STRUCTURE-TENSOR COMPONENTS}

All structure tensors necessary to calculate either $n$ GST to fourth, or the $n$ SGST to second order, are listed below. 
Due to the transversely isotropic nature of a rotationally symmetric fiber family, the following notation is used, where $f$ denotes the fiber direction while $n_{1}$ and $n_{2}$ express two arbitrary normal directions. Due to this symmetry, directions $n_{1}$ and $n_{2}$ can be used interchangeably. Further, it holds that all tensors listed below exert the highest degree of symmetry, meaning that they remain unchanged under any permutation of indices, wherefore only the amount of occurrences $(i, j, k)$ is accounted for, employing the style $\left(f^{i}, n_{1}^{j}, n_{2}^{k}\right)$. All components not listed are zero.

To further simplify notation, we define the $i$ th tensorial power of an arbitrary tensor $\mathbf{A}$ as

$$
\mathbf{A}^{(1)}=\underbrace{\mathbf{A} \otimes \ldots \otimes \mathbf{A}}_{i \text { times }} .
$$

\section{Fourth order}

The components have been derived previously by Pandolfi et al. [11] and are as follows:

$$
\begin{gathered}
\left\langle\mathbf{M}^{(2)}\right\rangle= \begin{cases}\frac{2 \kappa-\kappa_{2}}{2} & \hat{=}\left(f^{2}, n_{1}^{2}\right) \\
\frac{3 \kappa_{2}}{8} & \hat{=}\left(n_{1}^{4}\right) \\
\frac{\kappa_{2}}{8} & \hat{=}\left(n_{1}^{2}, n_{2}^{2}\right) .\end{cases} \\
\left\langle\mathbf{M}^{(3)}\right\rangle= \begin{cases}\text { 2. Sixth order } \\
\frac{1-6 \kappa+3 \kappa_{2}-\kappa_{3}}{\kappa_{3}} & \hat{=}\left(f^{6}\right) \\
\frac{\kappa_{2}-\kappa_{3}}{8} & \hat{=}\left(f^{4}, n_{1}^{2}\right) \\
\frac{5 \kappa_{3}}{16} & \hat{=}\left(f^{2}, n_{1}^{4}\right) \\
\frac{\kappa_{3}}{16} & \hat{=}\left(f^{2}, n_{1}^{2}, n_{2}^{2}\right)\end{cases}
\end{gathered}
$$

\section{Eighth order}

$$
\left\langle\mathbf{M}^{4}\right\rangle= \begin{cases}1-8 \kappa+6 \kappa_{2}-4 \kappa_{3}+\kappa_{4} & \hat{=}\left(f^{8}\right) \\ \frac{1}{2}\left(2 \kappa-3 \kappa_{2}+3 \kappa_{3}-\kappa_{4}\right) & \hat{=}\left(f^{6}, n_{1}^{2}\right) \\ \frac{3}{8}\left(\kappa_{4}-2 \kappa_{3}+\kappa_{2}\right) & \hat{=}\left(f^{4}, n_{1}^{4}\right) \\ \frac{1}{8}\left(\kappa_{4}-2 \kappa_{3}+\kappa_{2}\right) & \hat{=}\left(f^{4}, n_{1}^{2}, n_{2}^{2}\right) \\ \frac{5}{16}\left(\kappa_{3}-\kappa_{4}\right) & \hat{=}\left(f^{2}, n_{1}^{6}\right) \\ \frac{1}{16}\left(\kappa_{3}-\kappa_{4}\right) & \hat{=}\left(f^{2}, n_{1}^{4}, n_{2}^{2}\right) \\ \frac{35}{128} \kappa_{4} & \hat{=}\left(n_{1}^{8}\right) \\ \frac{5}{128} \kappa_{4} & \hat{=}\left(n_{1}^{6}, n_{2}^{2}\right) \\ \frac{3}{128} \kappa_{4} & \hat{=}\left(n_{1}^{4}, n_{2}^{4}\right) .\end{cases}
$$

\section{Different coefficients $\kappa_{i}$}

Generally, we define

$$
\kappa=\frac{1}{2}\left\langle\sin ^{2} \Theta\right\rangle \quad \text { and } \quad \kappa_{i}=\left\langle\sin ^{2 i} \Theta\right\rangle .
$$

It follows straight forward that

$$
\begin{aligned}
\kappa=\frac{1}{2}+\frac{1}{8 b_{\rho}}-\frac{1}{\sqrt{8 \pi b_{\rho}}} \frac{e^{2 b_{\rho}}}{\operatorname{erfi}\left(\sqrt{2 b_{\rho}}\right)} & \stackrel{b_{\rho} \rightarrow 0}{\longrightarrow} \frac{1}{3} \\
\kappa_{2}=\frac{3 \kappa-1}{2 b_{\rho}}+2 \kappa & \stackrel{b_{\rho} \rightarrow 0}{\longrightarrow} \frac{8}{15} \\
\kappa_{3}=\frac{5 \kappa_{2}-14 \kappa+2}{4 b_{\rho}}+2\left(\kappa_{2}-\kappa\right) & \stackrel{b_{\rho} \rightarrow 0}{\longrightarrow} \frac{16}{35} \\
\kappa_{4}=\frac{7 \kappa_{3}-16 \kappa_{2}+22 \kappa-2}{4 b_{\rho}} & \stackrel{b_{\rho} \rightarrow 0}{\longrightarrow} \frac{128}{315} .
\end{aligned}
$$

In the limit $b_{\rho} \rightarrow \infty$ all parameters converge to zero.

[1] P. Savadjiev, G. J. Strijkers, A. J. Bakermans, E. Piuze, S. W. Zucker, and K. Siddiqi, Heart wall myofibers are arranged in minimal surfaces to optimize organ function, Proc. Natl. Acad. Sci. 109, 9248 (2012).

[2] M. Amabili, P. Balasubramanian, I. Bozzo, I. D. Breslavsky, G. Ferrari, G. Franchini, F. Giovanniello, and C. Pogue, Nonlinear Dynamics of Human Aortas for Material Characterization, Phys. Rev. X 10, 011015 (2020). 
[3] V. Wang, P. Nielsen, and M. Nash, Image-based predictive modeling of heart mechanics, Annu. Rev. Biomed. Eng. 17, 351 (2015).

[4] P. Lamata, A. Cookson, and N. Smith, Clinical diagnostic biomarkers from the personalization of computational models of cardiac physiology, Ann. Biomed. Eng. 44, 46 (2016).

[5] M. C. Murphy, D. T. Jones, C. R. Jack, Jr., K. J. Glaser, M. L. Senjem, A. Manduca, J. P. Felmlee, R. E. Carter, R. L. Ehman, and J. Huston III, Regional brain stiffness changes across the Alzheimer's disease spectrum, NeuroImage: Clin. 10, 283 (2016).

[6] T. C. Gasser, R. W. Ogden, and G. A. Holzapfel, Hyperelastic modelling of arterial layers with distributed collagen fibre orientations, J. R. Soc. Interface 3, 15 (2006).

[7] K. Volokh, On arterial fiber dispersion and auxetic effect, J. Biomech. 61, 123 (2017).

[8] Y. Lanir, Constitutive equations for fibrous connective tissues, J. Biomech. 16, 1 (1983).

[9] G. A. Holzapfel and R. W. Ogden, On fiber dispersion models: Exclusion of compressed fibers and spurious model comparisons, J. Elast. 129, 49 (2017).

[10] Y. Lanir and R. Namani, Reliability of structure tensors in representing soft tissues structure, J. Mech. Behav. Biomed. Mater. 46, 222 (2015).

[11] A. Pandolfi and M. Vasta, Fiber distributed hyperelastic modeling of biological tissues, Mech. Mater. 44, 151 (2012).

[12] D. H. Cortes, S. P. Lake, J. A. Kadlowec, L. J. Soslowsky, and D. M. Elliott, Characterizing the mechanical contribution of fiber angular distribution in connective tissue: Comparison of two modeling approaches, Biomech. Model. Mechanobiol. 9, 651 (2010).

[13] G. A. Holzapfel, R. W. Ogden, and S. Sherifova, On fibre dispersion modelling of soft biological tissues: A review, Proc. R. Soc. A 475, 20180736 (2019).

[14] H. Gao, W. G. Li, L. Cai, C. Berry, and X. Y. Luo, Parameter estimation in a Holzapfel-Ogden law for healthy myocardium, J. Eng. Math. 95, 231 (2015).

[15] O. Gültekin, G. Sommer, and G. A. Holzapfel, An orthotropic viscoelastic model for the passive myocardium: Continuum basis and numerical treatment, Comput. Methods Biomech. Biomed. Eng. 19, 1647 (2016).

[16] G. A. Holzapfel and R. W. Ogden, Constitutive modelling of passive myocardium: A structurally based framework for material characterization, Philos. Trans. R. Soc. A: Math. Phys. Eng. Sci. 367, 3445 (2009).

[17] G. A. Holzapfel, T. C. Gasser, and R. W. Ogden, A new constitutive framework for arterial wall mechanics and a comparative study of material models, J. Elast. Phys. Sci. Solids 61, 1 (2000).

[18] J.-J. Hu, S. Baek, and J. Humphrey, Stress-strain behavior of the passive basilar artery in normotension and hypertension, J. Biomech. 40, 2559 (2007).

[19] G. Sommer, A. J. Schriefl, M. Andrä, M. Sacherer, C. Viertler, H. Wolinski, and G. A. Holzapfel, Biomechanical properties and microstructure of human ventricular myocardium, Acta Biomater. 24, 172 (2015).

[20] J. A. Niestrawska, C. Viertler, P. Regitnig, T. U. Cohnert, G. Sommer, and G. A. Holzapfel, Microstructure and mechanics of healthy and aneurysmatic abdominal aortas: Experimental analysis and modelling, J. R. Soc. Interface 13, 20160620 (2016).

[21] See Supplemental Material at http://link.aps.org/supple mental/10.1103/PhysRevApplied.13.064039 for MATLAB library comprising algorithms to compute stresses for the $n$ GST, $n$ SGST, and AI model.

[22] M. S. Sacks, Incorporation of experimentally-derived fiber orientation into a structural constitutive model for planar collagenous tissues, J. Biomech. Eng. 125, 280 (2003).

[23] T. S. Eriksson, A. J. Prassl, G. Plank, and G. A. Holzapfel, Modeling the dispersion in electromechanically coupled myocardium, Int. J. Numer. Methods Biomed. Eng. 29, 1267 (2013).

[24] A. Nikou, R. C. Gorman, and J. F. Wenk, Sensitivity of left ventricular mechanics to myofiber architecture: A finite element study, Proc. Inst. Mech. Eng., Part H: J. Eng. Med. 230, 594 (2016).

[25] H. M. Wang, H. Gao, X. Y. Luo, C. Berry, B. E. Griffith, R. W. Ogden, and T. J. Wang, Structure-based finite strain modelling of the human left ventricle in diastole, Int. J. Numer. Methods Biomed. Eng. 29, 83 (2013).

[26] M. T. Duong, Ph.D. thesis, Universitätsbibliothek der RWTH Aachen (2015).

[27] B. Baillargeon, N. Rebelo, D. D. Fox, R. L. Taylor, and E. Kuhl, The living heart project: A robust and integrative simulator for human heart function, Eur. J. Mech., A/Solids 48, 38 (2014).

[28] K. Li, R. W. Ogden, and G. A. Holzapfel, A discrete fibre dispersion method for excluding fibres under compression in the modelling of fibrous tissues, J. R. Soc. Interface 15, 20170766 (2018).

[29] H. Weisbecker, M. J. Unterberger, and G. A. Holzapfel, Constitutive modelling of arteries considering fibre recruitment and three-dimensional fibre distribution, J. R. Soc. Interface 12, 20150111 (2015).

[30] K. M. Hamdia, M. Marino, X. Zhuang, P. Wriggers, and T. Rabczuk, Sensitivity analysis for the mechanics of tendons and ligaments: Investigation on the effects of collagen structural properties via a multiscale modeling approach, Int. J. Numer. Methods Biomed. Eng. 35, e3209 (2019).

[31] S. P. Lake, D. H. Cortes, J. A. Kadlowec, L. J. Soslowsky, and D. M. Elliott, Evaluation of affine fiber kinematics in human supraspinatus tendon using quantitative projection plot analysis, Biomech. Model. Mechanobiol. 11, 197 (2012).

[32] G. A. Holzapfel and R. W. Ogden, On planar biaxial tests for anisotropic nonlinearly elastic solids. A continuum mechanical framework, Math. Mech. Solids 14, 474 (2009).

[33] A. J. Schriefl, G. Zeindlinger, D. M. Pierce, P. Regitnig, and G. A. Holzapfel, Determination of the layer-specific distributed collagen fibre orientations in human thoracic and abdominal aortas and common iliac arteries, J. R. Soc. Interface 9, 1275 (2011).

[34] A. V. Melnik, X. Luo, and R. W. Ogden, A generalised structure tensor model for the mixed invariant i8, Int. J. Non Linear Mech. 107, 137 (2018).

[35] G. M. Fomovsky, J. R. Macadangdang, G. Ailawadi, and J. W. Holmes, Model-based design of mechanical therapies 
for myocardial infarction, J. Cardiovasc. Transl. Res. 4, 82 (2011).

[36] G. M. Fomovsky, S. A. Clark, K. M. Parker, G. Ailawadi, and J. W. Holmes, Anisotropic reinforcement of acute anteroapical infarcts improves pump function, Circulation: Heart Failure 5, 515 (2012).

[37] W.-H. Zimmermann, I. Melnychenko, G. Wasmeier, M. Didié, H. Naito, U. Nixdorff, A. Hess, L. Budinsky, K. Brune, B. Michaelis, S. Dhein, A. Schwoerer, H. Ehmke, and T. Eschenhagen, Engineered heart tissue grafts improve systolic and diastolic function in infarcted rat hearts, Nat. Med. 12, 452 (2006).

[38] L. Ye, W.-H. Zimmermann, D. J. Garry, and J. Zhang, Patching the heart: Cardiac repair from within and outside, Circ. Res. 113, 922 (2013).
[39] P. Erhart, J. Roy, J.-P. P. de Vries, M. L. Liljeqvist, C. Grond-Ginsbach, A. Hyhlik-Dürr, and D. Böckler, Prediction of rupture sites in abdominal aortic aneurysms after finite element analysis, J. Endovasc. Ther. 23, 115 (2016).

[40] N. Maldonado, A. Kelly-Arnold, Y. Vengrenyuk, D. Laudier, J. T. Fallon, R. Virmani, L. Cardoso, and S. Weinbaum, A mechanistic analysis of the role of microcalcifications in atherosclerotic plaque stability: Potential implications for plaque rupture, Am. J. Physiol.: Heart Circ. Physiol. 303, H619 (2012).

[41] C. K. Kuo, J. E. Marturano, and R. S. Tuan, Novel strategies in tendon and ligament tissue engineering: Advanced biomaterials and regeneration motifs, BMC Sports Sci. Med. Rehabil. 2, 20 (2010). 